\title{
A high-order and fast scheme with variable time steps for the time-fractional Black-Scholes equation
}

\author{
Kerui Song ${ }^{1}$ and Pin Lyu ${ }^{1}$ \\ ${ }^{1}$ Southwestern University of Finance and Economics
}

October 30, 2021

\begin{abstract}
In this paper, a high-order and fast numerical method is investigated for the time-fractional Black-Scholes equation. In order to deal with the typical weak initial singularities of the solution, we construct a finite difference scheme with variable time steps, where the fractional derivative is approximated by the nonuniform Alikhanov formula and the sum-of-exponentials (SOE) technique. In the spatial direction, an average approximation with fourth-order accuracy is employed. The stability and the convergence with second-order in time and fourth-order in space of the proposed scheme are religiously derived by the energy method. Numerical examples are given to demonstrate the theoretical statement.
\end{abstract}

\section{Hosted file}

time_fractional_BS_MMAS.pdf available at https://authorea.com/users/443669/articles/543630a-high-order-and-fast-scheme-with-variable-time-steps-for-the-time-fractional-blackscholes-equation 